\section{ORIGINAL} RESEARCH

\author{
S.W. Hetts \\ J. Narvid \\ N. Sanai \\ M.T. Lawton \\ N. Gupta \\ H.J. Fullerton \\ C.F. Dowd \\ R.T. Higashida \\ V.V. Halbach
}

\title{
Intracranial Aneurysms in Childhood: 27-Year Single-Institution Experience
}

BACKGROUND AND PURPOSE: Pediatric aneurysms are rare and, thus, relatively poorly understood as compared to those in adults. Our aim was to characterize the clinical, imaging, treatment, and outcome data of patients younger than 19 years diagnosed with intracranial aneurysms at a tertiary care institution.

MATERIALS AND METHODS: We performed a retrospective medical record review of pediatric patients examined at our university hospital between 1981 and 2008.

RESULTS: We evaluated 77 patients (mean age, 12 years; 40 female, 37 male) with 103 intracranial aneurysms. Patients presented with headache $(45 \%)$, cranial neuropathies (16\%), nausea/vomiting $(15 \%)$, vision changes $(13 \%)$, trauma $(13 \%)$, seizure $(4 \%)$, or sensory changes $(3 \%)$. Subarachnoid hemorrhage occurred in 25 patients. Thirty-one fusiform aneurysms occurred in 25 patients. Fortyseven saccular aneurysms occurred in 35 patients. Twelve infectious aneurysms occurred in 6 patients. Fifteen traumatic aneurysms occurred in 12 patients. Fifty-nine patients underwent treatment of their aneurysms; 18 patients' conditions were managed conservatively. Nineteen patients underwent primary endovascular coiling, 1 patient had endovascular stent-assisted coiling, 11 patients underwent endovascular parent artery occlusion, 19 patients underwent surgical clipping, and 10 patients had aneurysms trapped and bypassed. Mortality was 1.3\%. Morbidity included $8 \%$ infarction and $4 \%$ new-onset seizures. Six patients developed new aneurysms or had enlargement of untreated aneurysms.

CONCLUSIONS: In our experience, intracranial aneurysms of childhood show a female predilection and predominantly saccular morphology. In neurovascular centers where microneurosurgical and endovascular options are available, most children with intracranial aneurysms can be successfully treated with low morbidity and mortality. Fusiform aneurysms require a combined microneurosurgical and endovascular approach more often than saccular aneurysms. The development of new aneurysms in pediatric patients during limited follow-up warrants further investigation.

$\mathbf{l}_{0}^{\mathrm{n}}$ ntracranial aneurysms are rare in childhood. Approximately $0.5 \%-4.6 \%$ of intracranial aneurysms occur in patients 18 years of age or younger. ${ }^{1-7}$ In a well-known study reported in 1966, only 41 of 6368 ruptured aneurysms were found in patients younger than 19 years. $^{8}$ Aneurysms in this population exhibit features that differ significantly from those in adults, including male predominance, a higher incidence of unusual anatomic locations such as the posterior circulation and internal carotid bifurcation, and greater numbers of giant aneurysms. ${ }^{1,9-12}$ Moreover, although some risk factors for the formation of intracranial aneurysms have been identified in adults (family history, age older than 50 years, smoking, cocaine use, and hypertension), in childhood most of these risk factors do not exist. ${ }^{13-16}$ In this context, some authors have advanced the concept of an aneurysmal vasculopathy involving vulnerable segments of the arterial tree. ${ }^{17-19}$

Not only do discrepancies exist in the clinical description of this rare entity but also in reports regarding its outcome after treatment. Good outcomes have been reported to range from $40 \%$ to $95 \%$ in the surgical literature. .,7,10,11,20-23 $^{\text {This hetero- }}$ geneity in outcomes represents patients treated during the

Received November 12, 2008; accepted after revision February 15, 2009.

From the Departments of Radiology (S.W.H., C.F.D., R.T.H., V.V.H.), Neurological Surgery (N.S., M.T.L., N.G.), and Neurology (H.J.F.), University of California, San Francisco, San Francisco, Calif; and Department of Radiology (J.N.), Stanford University, Palo Alto, Calif.

Please address correspondence to Steven W. Hetts, MD, 505 Parnassus Ave, L-352, San Francisco, CA 94143-0628; e-mail: steven.hetts@radiology.ucsf.edu

DOI 10.3174/ajnr.A1587 course of a half century, with considerable heterogeneity in patient characteristics, lesion characteristics, and operative technique. On the other hand, scientific literature pertaining to the endovascular management of aneurysms in childhood remains limited. The past 10 years have witnessed burgeoning enthusiasm for this approach and a gradual shift from traditional surgical approaches toward endovascular treatment at some centers. ${ }^{18,19,24}$ The increasing number of options available for the treatment of complex aneurysms found in the pediatric population requires data-driven planning by a multidisciplinary team. The purpose of the present article was to provide such data in the form of a single-center experience with this rare clinicopathologic entity.

\section{Materials and Methods}

\section{Subjects}

Between 1981 and 2008, 77 children with intracranial aneurysms not associated with arteriovenous malformations, dural arteriovenous fistulas, or vein of Galen malformations were treated at our institution (University of California San Francisco [UCSF] Medical Center and UCSF Children's Hospital). Medical records of these patients were retrospectively reviewed, and clinical history, neurologic examination, and diagnostic work-up were extracted. Patients were evaluated in clinics attended by neurosurgeons, neurologists, and interventional neuroradiologists. Delayed follow-up information was obtained by a review of the most recent outpatient clinic visit and a telephone questionnaire. In case of death occurring outside our institution, functional status before death was determined by discussion with family members. 


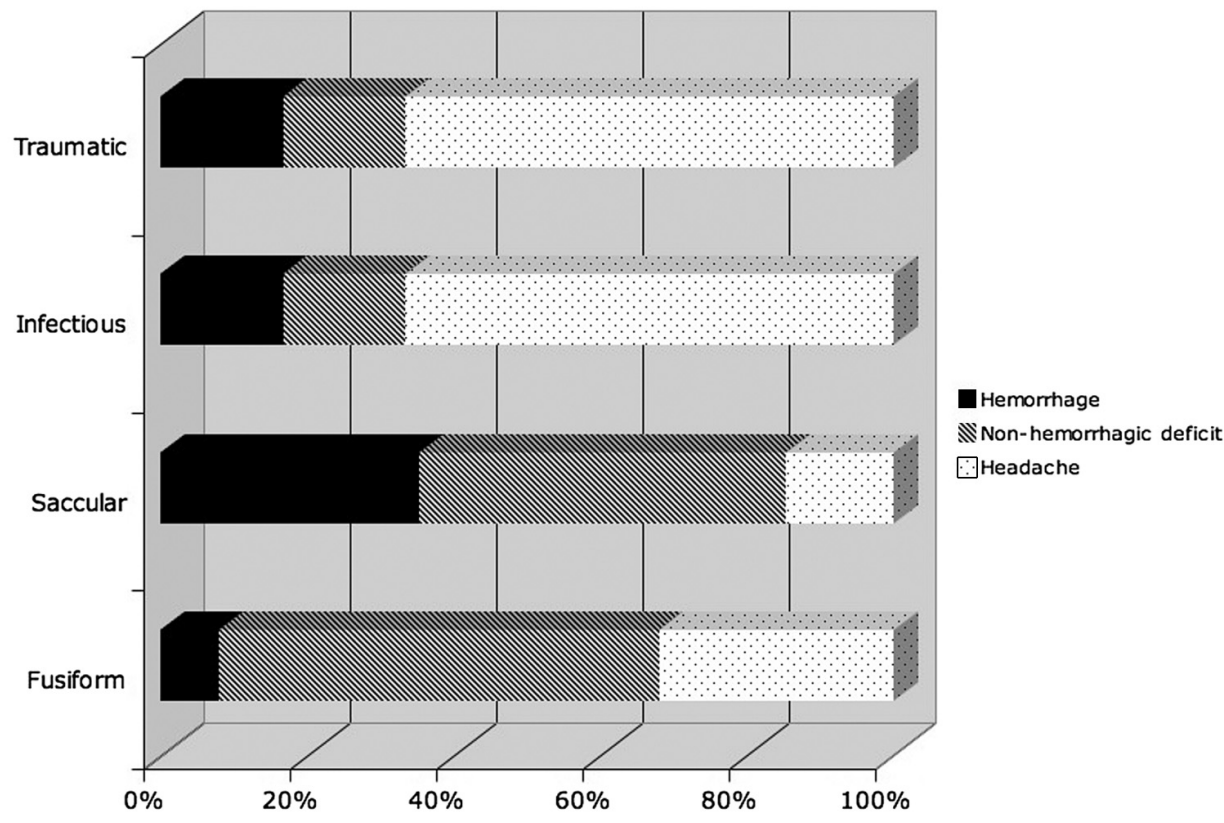

Fig 1. Clinical symptoms at presentation in relation to the type of aneurysm (percentages).

\section{Diagnostic Imaging}

Pre- and postintervention CT or MR images and angiograms or both were obtained, and dictated reports were reviewed. A diagnosis of subarachnoid hemorrhage (SAH) was confirmed with CT findings of subarachnoid blood in the basal cisterns or with analysis of CSF obtained by lumbar puncture. In all such cases, early intervention was performed. Posttreatment angiography was performed either intraoperatively or during the immediate postoperative hospitalization period, when possible. Aneurysms were classified by using the methods of Lasjaunias et $\mathrm{al}^{18}$ into the following 4 groups on the basis of angiographic morphology and clinical context: 1) fusiform aneurysms without clinical association of infection or trauma; 2) saccular aneurysms without clinical association of infection or trauma; 3 ) infectious aneurysms in cases of associated systemic infection or immunocompromise; and 4) traumatic aneurysms in patients with associated significant trauma.

\section{Treatment}

Subsequent to angiographic diagnosis, patients were treated by surgery, endovascular intervention, a combination of the 2, or observation. Endovascular treatment by interventional neuroradiologists included the use of detachable coils with or without stents delivered through microcatheters after transfemoral superselective catheterization of the involved intracranial arteritis. Alternatively, in the case of aneurysms not amenable to coil obliteration with preservation of the parent artery, parent artery occlusion via detachable balloons or coils was performed after demonstration of adequate collateral circulation (angiographically and, when possible, by awake balloon test occlusion). If a significant aneurysm neck remnant was detected on follow-up surveillance angiography, retreatment was performed with further endovascular coiling and/or stent placement. Surgical treatment consisted of surgical clipping or bypass trapping performed by cerebrovascular neurosurgeons.

\section{Follow-Up}

Clinical follow-up was performed by neurologists, interventional neuroradiologists, and/or neurosurgeons, depending on the treatment technique. The patients in the untreated observation group un- derwent annual follow-up MR imaging studies to evaluate aneurysmal growth and stability. Patients in the endovascular group were followed with yearly MR imaging and clinical examinations; in cases of new imaging or clinical findings, angiography was performed. Surgical patients were followed clinically in the neurosurgical outpatient clinic and with imaging when clinically indicated due to new symptoms or at 5 years following initial treatment, whichever came first.

\section{Results}

\section{Baseline Aneurysm Characteristics}

There were 40 females (52\%) and 37 (48\%) males who ranged in age (at diagnosis) from 3 months to 18 years (mean, $12 \pm 5$ years). There were a total of 103 aneurysms, $11 \%$ of which measured $>25 \mathrm{~mm}$. Thirty-one fusiform aneurysms occurred in 25 patients. Forty-seven saccular aneurysms occurred in 35 patients. Twelve infectious aneurysms occurred in 6 patients. Fifteen traumatic aneurysms occurred in 12 patients. At the time of initial presentation, patients reported headache (45\%), cranial neuropathies (16\%), nausea/vomiting (15\%), vision changes $(13 \%)$, trauma $(13 \%)$, seizure $(4 \%)$, and sensory changes (3\%). Twenty-five patients (32\%) presented with $\mathrm{SAH}$. Although 4 patients had documented intracranial vasospasm, only 1 required treatment due to progressive neurologic deficit. The rate of hemorrhagic presentation differed considerably among aneurysm subtypes (Fig 1). Hemorrhage was twice as likely to result from saccular aneurysm rupture than from fusiform or infectious aneurysms, occurring in $>35 \%$ of cases. Two cases of SAH among the traumatic aneurysms were found. Six patients had aneurysms discovered incidentally during imaging studies obtained for unrelated indications. Whereas 34 patients had identifiable comorbidities that might be etiologically related to aneurysm formation (Table 1), the remaining 43 patients had no such conditions.

Type I Noninfectious Nontraumatic Fusiform Aneurysms Most fusiform aneurysms occurred in the anterior circulation (24/31 aneurysms, 77\%). There were 11 intradural internal 


\begin{tabular}{|c|c|}
\hline Category & Specific Comorbidity \\
\hline Traumatic & $\begin{array}{l}\text { Motor vehicle crash (3), Pedestrian-versus- } \\
\text { automobile accident with skull base } \\
\text { fractures, prior gunshot wound (2), prior } \\
\text { skateboard accident, fall, head trauma } \\
\text { not otherwise specified }\end{array}$ \\
\hline Ischemic & $\begin{array}{l}\text { Prior insular infarct, prior external-carotid- } \\
\text { to-internal carotid artery bypass } \\
\text { grafting (2) }\end{array}$ \\
\hline Vasculopathic & Fibromuscular dysplasia \\
\hline Dermatologic & $\begin{array}{l}\text { PHACE, facial and retinal hemangiomas, } \\
\text { vascular birthmark }\end{array}$ \\
\hline Hormonal & $\begin{array}{l}\text { Growth hormone deficiency with } \\
\text { neuromigrational disorder and } \\
\text { Moyamoya syndrome, Seckel syndrome } \\
\text { (growth hormone deficiency), Majewski } \\
\text { type II dwarfism }\end{array}$ \\
\hline Hematologic & Von Willebrand disease \\
\hline Immunologic & $\begin{array}{l}\text { AIDS, congenital immunodeficiency, } \\
\text { combined immunodeficiency syndrome } \\
\text { with cerebral angiodysplasia }\end{array}$ \\
\hline Cardiovascular & $\begin{array}{l}\text { Congenital aortic stenosis with } \\
\text { endocarditis, tricuspid atresia with } \\
\text { Fontan procedure, patent ductus } \\
\text { arteriosus }\end{array}$ \\
\hline Neoplastic & Juvenile pilocytic astrocytoma \\
\hline Neurologic & Seizure disorder \\
\hline Other syndromic & $\begin{array}{l}\text { Tuberous sclerosis (2), autosomal } \\
\text { dominant polycystic kidney disease, } \\
\text { trisomy } 21 \text { with endocarditis, } \\
\text { hemiatrophy, mitochondrial disorder } \\
\text { with optic atrophy, family history of } \\
\text { Marfan syndrome }\end{array}$ \\
\hline
\end{tabular}

* Numbers in parentheses indicate comorbidities present in multiple patients. Note that infection is not listed as a comorbidity; however, patients with immunodeficiency syndromes and patients with endocarditis due to an identified underlying risk factor (eg, congenital aortic stenosis) are listed under their primary comorbid condition.

carotid artery (ICA) aneurysms, 7 middle cerebral artery (MCA) aneurysms, 3 extradural ICA aneurysms, 3 anterior cerebral artery (ACA) aneurysms, and 7 aneurysms of the posterior circulation (Figs 2 and 3). The mean maximal aneurysm diameter was $15.4 \pm 9 \mathrm{~mm}$. Patients harbored an average of $1.2 \pm 0.6$ aneurysms, with a range of $1-3$ aneurysms. The mean age of presentation with a fusiform aneurysm was $11.8 \pm 5.5$ years. The male-female ratio was $12: 13$. There were 6 children with congenital diseases associated with fusiform aneurysms. One child had aortic anomalies (possibly PHACE syndrome), 1 child had a facial hemangioma, 1 child had congenital hemiatrophy, 1 child had a patent ductus arteriosus, 1 child had a mitochondrial disorder, and another had tuberous sclerosis. One teenager admitted to cocaine and amphetamine abuse at the time of diagnosis. Of all aneurysm subtypes, fusiform aneurysms received the most heterogeneous management strategies. Endovascularly, 7 patients in this group received aneurysm coiling, and 4 received balloon occlusion of the involved ICA. Surgically, 2 were clipped, 1 was clip occluded, and 4 were bypass/trapped. Nine patients received no intervention besides observation and close clinical follow-up. Mean follow-up in all patients with fusiform aneurysms was 113 months.

\section{Type II Noninfectious Nontraumatic Saccular Aneurysms}

Most saccular aneurysms were also located in the anterior circulation (37/47 aneurysms, 79\%). There were 22 intradural ICA aneurysms, 7 MCA aneurysms, 4 extradural ICA aneurysms, 4 ACA aneurysms, and 10 aneurysms of the posterior circulation (Figs 2 and 3). The mean maximal aneurysm diameter in this group was $10.2 \pm 11 \mathrm{~mm}$. Patients harbored an average of $1.3 \pm 1.5$ saccular aneurysms with a range of $1-10$ aneurysms. The mean age of presentation with a saccular aneurysm was $12.9 \pm 4.9$ years. The male-female ratio was 20:15. Eight patients had other associated conditions. Two patients had congenital growth hormone dysregulation syndromes (Seckel syndrome and Majewski osteodysplastic primordial dwarfism type II), 1 patient had congenital heart disease (tricuspid atresia status post-Fontan procedure), 1 patient had tuberous sclerosis; 1 patient had a neuromigrational disorder, 1 patient had a juvenile pilocytic astrocytoma, and another patient had polycystic kidney disease. One child had a relative with Marfan disease. Among saccular aneurysms, 11 were coiled, 1 was stent coiled, 3 underwent balloon occlusion, 22 were clipped, 2 underwent combination endovascular and surgical treatment, and 7 were observed. Mean follow-up on all patients with saccular aneurysms was 79 months.

\section{Type III Infectious Aneurysms}

Infectious aneurysms were located in the anterior circulation $92 \%$ of the time (11/12 aneurysms). There were 3 intradural ICA aneurysms; 3 MCA, 1 extradural ICA, 4 ACA aneurysms; and 1 basilar tip aneurysm (Figs 2 and 3). Mean aneurysm size was $10.7 \mathrm{~mm}$. Patients harbored an average of $2 \pm 1.3$ mycotic aneurysms with a range of $1-4$ aneurysms. The mean age of presentation with an infectious aneurysm was $9 \pm 3.5$ years. The male-female ratio was 5:1. Associated infections and/or immunodeficiencies included 2 children with congenital immunodeficiencies, 1 child with acquired immunodeficiency syndrome (AIDS), 2 with endocarditis, and 1 with meningoencephalitis. One child with endocarditis additionally had trisomy 21 . Three of these children were surgically treated with trapping and bypass. The aneurysm of 1 patient was coiled. Two children received follow-up and observation. All patients received appropriate systemic antibiotic therapy in addition to any surgical or endovascular intervention. Mean follow-up in all patients with infectious aneurysms was 14 months.

\section{Type IV Traumatic Aneurysms}

Traumatic aneurysms were also predominantly within the anterior circulation (10/15 aneurysms, 67\%). There were 5 intradural ICA, 1 MCA, 5 extradural ICA, and 4 posterior circulation aneurysms (Figs 2 and 3). As would be expected on the basis of the mechanism of trauma, aneurysms were more common near the skull base than in the supratentorium. Patients harbored an average of $1.3 \pm 0.6$ traumatic aneurysms with a range of 1-3 aneurysms. The mean age of presentation with a traumatic aneurysm was $12.7 \pm 4.4$ years. The male-female ratio was 7:5. Six aneurysms underwent balloon occlusion, 3 were coiled, 1 was clipped, 1 was trapped and resected, and 4 were observed. Mean follow-up in all patients with traumatic aneurysms was 58 months. 


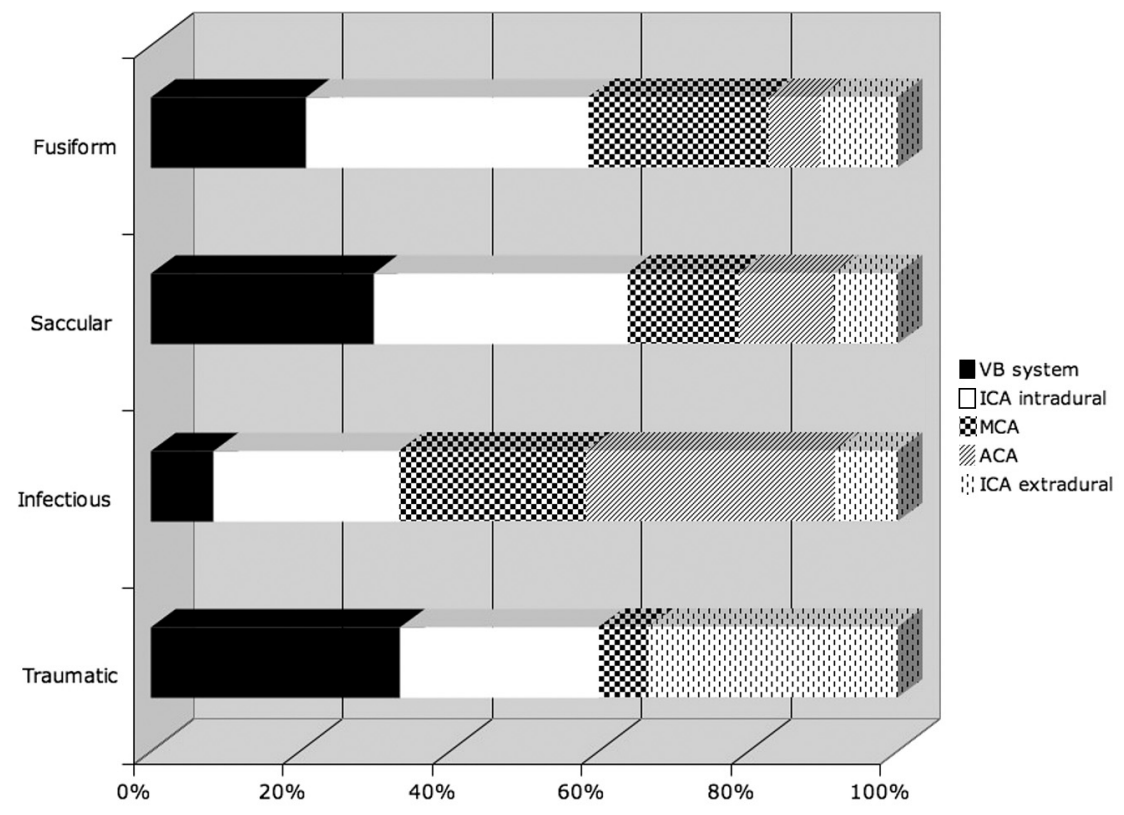

Fig 2. Vascular location of 103 aneurysms in relation to their type (percentages).

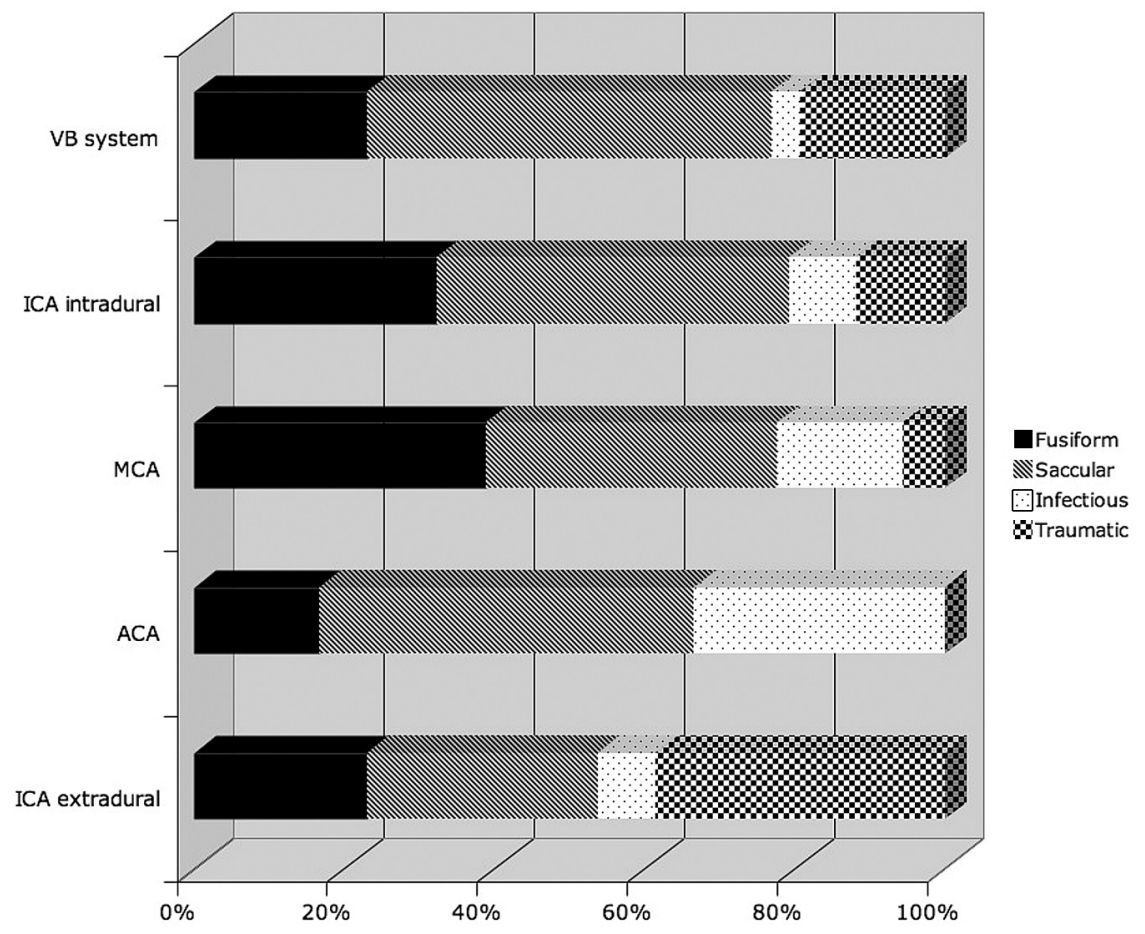

Fig 3. Aneurysm subtype expressed in relation to anatomic location (percentages).

\section{Aneurysm Management}

A multidisciplinary team including neurologists, cerebrovascular neurosurgeons, and neurointerventional radiologists made management decisions for individual patients. Combined approaches were used when 1 technique of treatment could not achieve ideal results. A total of 59 patients underwent treatment of their aneurysms; 18 patients were managed conservatively with close follow-up and serial imaging (Fig 4). None of the 18 patients undergoing conservative follow-up had aneurysm rupture during the time of observation, though
1 patient did have asymptomatic aneurysm enlargement prompting stent-assisted coiling.

\section{Selective Coiling}

Nineteen patients underwent primary aneurysm coiling. The mean age was $11 \pm 6$ years. The mean maximal aneurysm diameter was $13 \pm 12 \mathrm{~mm}$. Six patients had SAH at presentation. There was 1 case of intraprocedural arterial puncture by a guidewire in the first decade of this series; this event was complicated by a partial ACA infarction and concomitant 


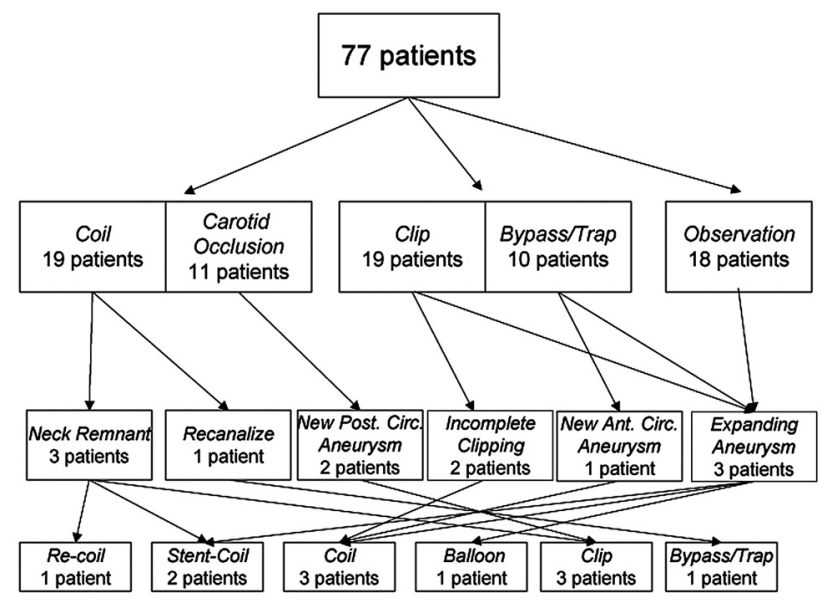

Fig 4. Clinical management and treatment procedures in 77 children with intracranial aneurysms.

lower extremity monoparesis. One patient had intraprocedural aneurysm microrupture, which was immediately sealed with heparin reversal and placement of additional coils; no clinical deficit was evident on the patient's waking from the procedure. The average follow-up period was 23 months with 1 patient lost to follow-up. Four patients required retreatment. Three of these were due to aneurysmal neck remnants identified at follow-up angiography. Retreatment included placement of additional detachable coils in a fusiform posterior cerebral artery (PCA) aneurysm, the use of an intracranial stent with placement of additional detachable coils in a traumatic MCA M1 segment aneurysm, surgical bypass trapping for a fusiform M1 MCA segment aneurysm, and surgical clipping of an ICA terminus aneurysm, which developed partial recanalization 6 years following initial coiling. A child with multiple fusiform aneurysms associated with AIDS was treated with endovascular coiling. Twenty months later, this patient developed a de novo vertebrobasilar aneurysm, which presented with fatal rupture, producing the only known death in this study population.

\section{Endovascular Parent Artery Occlusion}

Eleven patients underwent endovascular balloon occlusion of either extensive fusiform aneurysms or the parent vessels of large saccular aneurysms between 1987 and 2003. The mean patient age was $14 \pm 3$ years. The mean maximal aneurysm diameter was $25 \pm 6 \mathrm{~mm}$. One patient had SAH at presentation. Intraprocedural and periprocedural complications included the following: 1 transient hemiparesis, 1 transient monocular vision loss, and 1 transient sixth-nerve palsy and trigeminal sensory loss. The average follow-up period was 26 months, with no patients lost to follow-up. Two patients required retreatment due to de novo posterior circulation aneurysms. Both had fusiform aneurysms of the ICA at initial diagnosis. Both were evaluated by a cerebrovascular neurosurgeon at an outside institution and underwent surgical bypass and clipping. One patient had a brain stem infarct at surgery; the other patient did well during the follow-up period. Another patient, also with a fusiform aneurysm of the petrous ICA, developed a new posterior circulation aneurysm, which was deemed untreatable. Three years after diagnosis, the vertebrobasilar aneurysm remained stable.

\section{Selective Clipping}

Nineteen patients underwent primary surgical clipping. The mean age of this group was $12 \pm 6$ years. The mean maximal aneurysm diameter was $12 \pm 10 \mathrm{~mm}$. Thirteen patients in this group presented with SAH. Periprocedural complications included the following: 1 transient third nerve palsy, 1 transient hemiparesis, 1 MCA occlusion with concomitant infarct and hemiparesis, 1 worsened lower extremity monoparesis, and 1 basilar trunk occlusion with brain stem/thalamic infarction and concomitant hemiparesis, ophthalmoplegia, and mutism. One case of endoventricular drain-associated ventriculitis also occurred in this group. One patient developed a scalp arteriovenous fistula and seizure disorder 4 years after initial presentation. The mean follow-up period was 23 months, but follow-up was less consistent in this group, with 9 patients having $<1$ year of follow-up. A patient who had previously undergone partial clipping of a proximal PCA fusiform aneurysm at an outside institution, which was complicated by thalamic infarction, underwent successful coil embolization of a growing aneurysm remnant without complication. One patient with SAH and Majewski osteodysplastic primordial dwarfism type II had 10 intracranial aneurysms, half of which were treated with unilateral craniotomy and clipping and 1 of which (a contralateral P2 PCA aneurysm) underwent coiling during the same hospitalization for the acute condition. At 6-month surveillance angiography, an enlarging posterior communicating artery aneurysm (PcomA) was also coiled in this patient.

\section{Surgical Trapping}

Aneurysms in 10 patients were trapped and/or bypassed. The mean age of this group was $12 \pm 4$ years. The mean maximal aneurysm diameter was $12 \pm 9 \mathrm{~mm}$. Three patients presented with SAH. Periprocedural complications included the following: 1 transient sixth-nerve palsy, 1 case of diabetes insipidus with delayed vision loss, 1 case of transient hemiparesis with persistent facial palsy, and 1 case of mild hemiparesis. Additionally, there was 1 case of ventriculitis in this group, and 2 patients developed seizure disorders postoperatively. One patient developed a chronic subdural hematoma, which required surgical drainage 6 months after the bypass-trapping procedure. Follow-up in this group was the longest, with an average of 87 months, though 1 patient was only followed for 1 month. Two patients required aneurysm retreatment. One patient with a giant saccular aneurysm of the MCA underwent attempted surgical trapping, which was incompletely successful due to diffuse arterial vasospasm. Subsequent angiography showed $70 \%$ occlusion with persistent filling of the aneurysm dome. Detachable balloon embolization was complicated by MCA infarction and hemiparesis. Another patient with multiple fusiform aneurysms of the right MCA and ICA developed a de novo aneurysm of the ACA 12 years after bypass/trapping of his initial aneurysms. The new aneurysm was successfully coiled without complication.

\section{Conservative Observation}

Eighteen patients with long-segment vascular dysplasia involving multiple territories were managed conservatively. None of these patients presented with SAH. The mean age was $11 \pm 5$ years. The mean maximal aneurysm diameter was $6 \pm$ $3 \mathrm{~mm}$. Follow-up length was an average of 41 months, with 1 

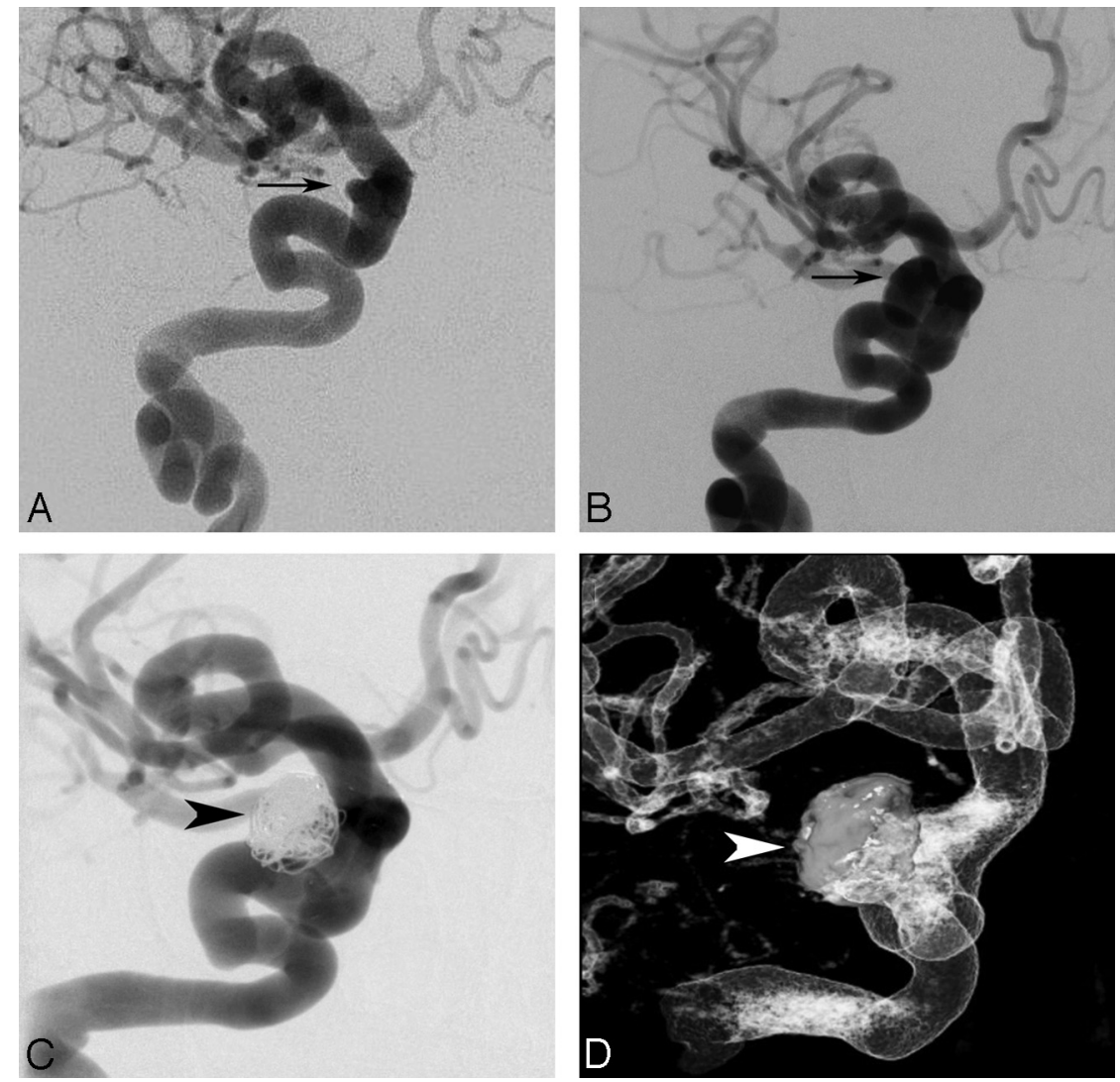

Fig 5. Progressive enlargement of a saccular paraophthalmic aneurysm superimposed on fusiform internal carotid vasculopathy. $A$, Right ICA angiogram of a patient at age 10 , anteroposterior projection demonstrating a tortuous ICA with a superimposed 3-mm saccular paraophthalmic aneurysm (arrow). The patient underwent conservative observation with annual MR angiography which, 4 years later, suggested aneurysm progression. B, Right ICA angiogram of a patient at age 14, anteroposterior projection, demonstrating interval enlargement of the paraophthalmic aneurysm (arrow) to $13 \mathrm{~mm}$, prompting a decision in favor of treatment. $C$ and $D$, Right ICA angiogram, anteroposterior projection $(C)$, and $3 D$ rotational angiogram (D) of patient at age 14 immediately following successful stent-assisted coiling of the paraophthalmic aneurysm (arrowheads indicate coil mass).

\begin{tabular}{|c|c|c|c|c|c|c|c|}
\hline $\begin{array}{l}\text { Age at First } \\
\text { Presentation } \\
\text { (yr) }\end{array}$ & Sex & Index Aneurysm & First Treatment & $\begin{array}{c}\text { Time to } \\
\text { De Novo or } \\
\text { Enlarging } \\
\text { Aneurysm }\end{array}$ & $\begin{array}{c}\text { De Novo or } \\
\text { Enlarging } \\
\text { Aneurysm } \\
\text { Location }\end{array}$ & Clinical Result & Comorbidity \\
\hline 11 & $M$ & ICA fusiform & ICA balloon occlusion & $3 y r$ & VB junction fusiform & Successful clipping & Vascular birthmark \\
\hline 7 & M & ICA fusiform & $\begin{array}{l}\text { ICA balloon-coil } \\
\text { occlusion }\end{array}$ & $3 \mathrm{yr}$ & $\begin{array}{l}\text { Intracranial vertebral } \\
\text { fusiform }\end{array}$ & Untreatable & Hemiatrophy \\
\hline 15 & $\mathrm{~F}$ & $\begin{array}{l}\text { ICA, MCA, ACA } \\
\text { fusiform giant }\end{array}$ & Bypass, trapping & $12 \mathrm{yr}$ & AcomA fusiform & $\begin{array}{l}\text { Wrapping, completion } \\
\text { coiling }\end{array}$ & None \\
\hline 8 & M & ACA giant mycotic & Coiling & $20 \mathrm{mo}$ & VB fusiform & Died from SAH & AIDS \\
\hline 17 & $\mathrm{~F}$ & 10 saccular aneurysms & $\begin{array}{l}\text { Clip } 6 \text { ipsilateral; coil } \\
1 \text { contralateral }\end{array}$ & $6 \mathrm{mo}$ & $\begin{array}{l}\text { Enlarging saccular } \\
\text { PcomA }\end{array}$ & Successful coiling & $\begin{array}{l}\text { Majewski type II } \\
\text { dwarfism }\end{array}$ \\
\hline 10 & $\mathrm{~F}$ & $\begin{array}{l}\text { Long-segment fusiform } \\
\text { ICA }\end{array}$ & Observation & $4 \mathrm{yr}$ & $\begin{array}{l}\text { Enlarging saccular } \\
\text { ICA aneurysm }\end{array}$ & Successful stent coiling & $\begin{array}{l}\text { Tricuspid atresia after } \\
\text { Fontan procedure }\end{array}$ \\
\hline
\end{tabular}

Note:-VB indicates vertebrobasilar; ICA, internal carotid artery; MCA, middle cerebral artery; ACA, anterior cerebral artery; AcomA, anterior communicating artery; PcomA, posterior communicating artery; SAH, subarachnoid hemorrhage.

patient lost to follow-up 1 month after initial evaluation and the remaining 17 patients observed for between 6 months and 10 years. These patients underwent serial MR imaging studies to follow aneurysm growth. Two patients showed minimal aneurysm enlargement. One patient had significant enlargement of a paraophthalmic saccular aneurysm superimposed on a highly dysplastic ICA and underwent primary stent-assisted coiling 4 years into the observation period (Fig 5). No other patients required treatment. None of these patients had SAH during the follow-up period.

\section{De Novo and Enlarging Aneurysms}

Four patients developed new aneurysms, and 2 additional patients had significant enlargement of untreated aneurysms while under surveillance following index aneurysm treatment or while undergoing conservative observation (Table 2). New aneurysms occurred an average of $4.9 \pm 4.8$ years after initial aneurysm treatment. Enlarging untreated aneurysms were identified in 1 patient 6 months after index aneurysm treatment and in another patient 4 years into conservative observation. 
Patients with de novo or enlarging untreated aneurysms came from several subgroups in our study. Two patients had undergone prior ICA occlusion (2/11 or $18 \%$ of the carotid artery occlusion subgroup), and both developed fusiform vertebral or vertebrobasilar aneurysms 3 years after carotid takedown. One patient had received right common-carotid-tocortical right MCA bypass with trapping of right ACA, MCA, and ICA fusiform aneurysms (1/10 or $10 \%$ of the bypass/trap subgroup) and 12 years later developed a fusiform anterior communicating artery (AcomA) aneurysm, which then was treated with surgical wrapping and, after 2 additional years of aneurysm growth, completion coiling. One patient with AIDS, whose infectious giant ACA aneurysm was coiled, re-presented 20 months later with fatal SAH from a new vertebrobasilar aneurysm; this was the only death in the series. One patient with Majewski type II dwarfism initially had 6 leftsided aneurysms clipped and 1 right-sided P1/2 PCA aneurysm coiled during the same hospitalization for SAH. Six months later, this patient had significant enlargement of a right PcomA saccular aneurysm, which was then coiled. One patient from the conservative observation group, 4 years into observation, had significant enlargement of a saccular paraophthalmic ICA aneurysm superimposed on long-segment fusiform dilation of the cervical-through-intracranial ICA. The saccular paraophthalmic component of the aneurysm was successfully stent coiled (Fig 5).

\section{Evolution of Aneurysm Management with Time}

Since 1981, there has been an evolution in microneurosurgical and endovascular techniques used at our hospital. Separating our cohort into discrete time periods underscores the changes in aneurysm management that have occurred. Between 1981 and 1985, 100\% of cases were surgical (clipping or bypass/ trapping). From 1986 to $1990,18 \%$ of cases were treated surgically, 73\% were treated endovascularly (all being parent artery occlusions), and 9\% were observed. Primary endovascular coiling of pediatric aneurysms at UCSF began in 1991; during the next 5 years, $33 \%$ of cases were treated surgically, $47 \%$ were treated endovascularly (57\% by coiling and $43 \%$ by parent artery occlusion), and 20\% were observed. From 1996 to 2000 , $27 \%$ of cases were surgical, $33 \%$ were endovascular (all coiling), and $40 \%$ were observed. Since $2001,45 \%$ of cases were treated surgically, $45 \%$ were treated endovascularly (14\% parent artery occlusion and $86 \%$ coiling or stent-assisted coiling), and 10\% were observed. As demonstrated in Fig 4, extensive crossover between treatment groups has occurred.

\section{Discussion}

Intracranial aneurysms in children differ from those in adults in location, morphology, etiology, natural history, and, hence, management. This study reports the experience of a single university hospital that has long benefited from the collaboration between cerebrovascular neurosurgeons, pediatric neurosurgeons, interventional neuroradiologists, and pediatric neurologists.

Several series have been published recently detailing the experience of various institutions with pediatric intracranial aneurysms, ${ }^{18,20,22}$ including 1 from our institution, ${ }^{23}$ which included patients with intracranial aneurysms treated between 1977 and 2003 but did not include extradural intracranial (ie, internal carotid petrous and cavernous segment) aneurysms. Inclusion of aneurysms of the petrocavernous carotid artery is relevant because several patients were noted to have longsegment vascular dysplasias, which involved both intradural and extradural segments of craniocervical arteries. This is in keeping with the notion that a genetic or epigenetic field defect may lead to diffuse vasculopathies of multiple arteries in some patients. Children are not simply small adults; their aneurysms tend to be larger, have more complex shapes, and are more likely to be located in the posterior circulation as compared with aneurysms in adults.

As previously described, ${ }^{18}$ aneurysm morphology and etiology can be separated into 4 categories: infectious (often termed "mycotic" regardless of the inciting organism), posttraumatic (which can have a variety of appearances but must be associated with documented significant trauma), nontraumatic noninfectious fusiform, and nontraumatic noninfectious saccular. In the series of Lasjaunias et al, ${ }^{18}$ a majority of aneurysms were nontraumatic noninfectious fusiform, whereas in the current series, only a third of aneurysms were. Aneurysm morphology and etiology have therapeutic implications. The proportion of patients with fusiform aneurysms presenting with rupture in the current series is lower $(2 / 25$ or $8 \%)$ than that in the series of Lasjaunias et al (40\%). In both series, saccular aneurysms were the most likely to rupture ( $>75 \%$ in the series of Lasjaunias et al and $>35 \%$ in the current series). Infectious aneurysms in both series ruptured at a relatively low rate $(17 \%$ in our series and $13 \%$ in the prior series). Most interesting, 5 of the 6 patients with infectious aneurysms were male and presented with endocarditis, and all had immunodeficiency (AIDS, severe combined immunodeficiency, and congenital immunodeficiency) or structural cardiac anomalies (aortic stenosis, trisomy 21 with endocardial cushion defect).

X-linked immunodeficiencies have previously been reported to be associated with intracranial aneurysm formation, as has AIDS. ${ }^{25}$ The sole female with an infectious aneurysm developed a cavernous ICA aneurysm in the setting of meningitis and had no known immunologic or cardiac predisposition to infectious aneurysm formation. The number of traumatic aneurysms in both series was low ( 2 in the series of Lasjaunias et al, ${ }^{18}$ and 11 in the current series). One traumatic aneurysm ruptured in the prior series and in the current series. Selection bias may be present in the current series in that our university hospital is not the regional trauma center. Although patients may acquire intracranial aneurysms traumatically, these aneurysms may rupture and result in death before hospitalization or transfer to a tertiary care center. Alternatively, intrinsically normal traumatized blood vessels may have a better natural history than intrinsically abnormal blood vessels that develop fusiform or saccular aneurysms.

The presence of multiple synchronous or metachronous aneurysms implies an underlying vasculopathy or predilection to aneurysm formation. Among the most challenging cases in our series are those in which a diffuse vasculopathy results in long-segment fusiform dilation of an artery. As a case in point (Fig 6), 3 years after balloon occlusion of an affected ICA in an 11-year-old boy, de novo fusiform dilation of the vertebrobasilar junction developed. In this particular case, redirection of flow following carotid occlusion, with increased velocities 


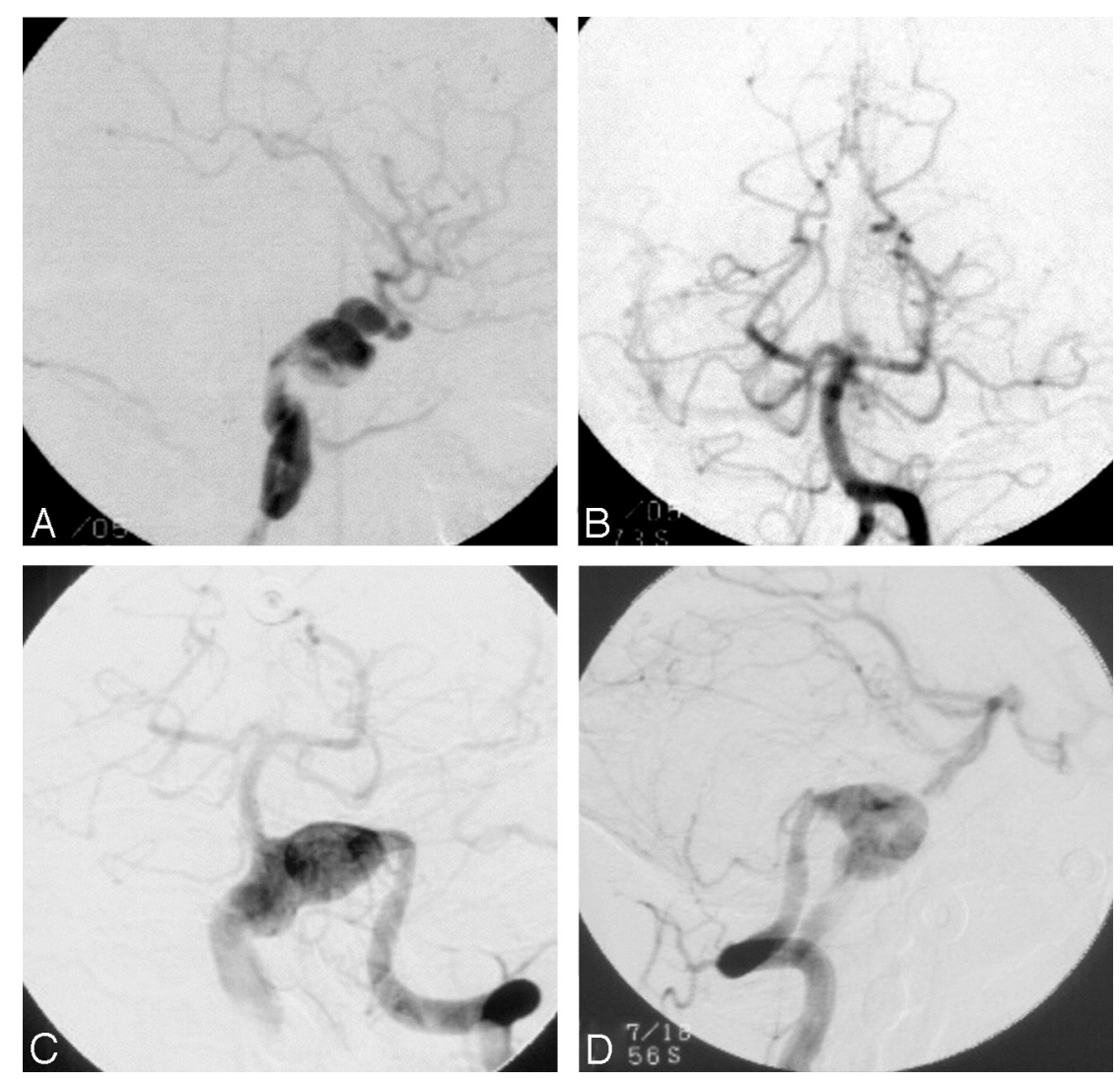

Fig 6. Development of a de novo fusiform vertebrobasilar junction aneurysm 3 years after treatment of a fusiform ICA aneurysm with balloon takedown of the ICA in an adolescent male patient. $A$, Left ICA angiogram of the patient at age 11, lateral projection. $B$, Vertebrobasilar angiogram at age 11 , anteroposterior projection. The left ICA was successfully occluded with 2 detachable balloons immediately following the angiograms shown in $A$ and $B$. Three years later an MR angiogram (not shown) suggested a new aneurysm at the vertebrobasilar junction. Conventional angiography was performed, confirming this diagnosis $(C$ and $D)$. C Vertebrobasilar angiogram at age 14 , anteroposteror projection. $D$, Vertebrobasilar angiogram at age 14 , lateral projection.

in the posterior circulation, might have contributed to the development of a second aneurysm. Alternatively, the natural history of such a diffuse vasculopathy might be the development of multiple fusiform aneurysms. Such patients have contributed to consideration of watchful waiting as a management strategy for particularly extensive cases of vasculopathy.

Of the 18 patients managed with observation in our series, 12 had extensive fusiform aneurysms and none experienced $\mathrm{SAH}$ during the follow-up period. However, given our experience of de novo posterior circulation aneurysms arising in patients previously receiving carotid occlusion therapy for fusiform ICA aneurysms (Fig 6), possibly though not definitively due to vascular flow alterations, we currently make a greater effort to preserve parent artery flow, even if this requires stent-assisted coiling (Fig 5) or other more extensive surgery. Regardless of aneurysm treatment or observation, children with intracranial aneurysms require follow-up imaging and clinical surveillance, given their expected long life spans during which additional aneurysms could arise or treated aneurysms could recur. As suggested previously, ${ }^{18}$ aneurysmal disease is both an acute and chronic condition and requires longitudinal management, with emphasis on the ongoing disease entity itself as opposed to just the mode of treatment when an aneurysm becomes acutely symptomatic. The fact that 4 patients in the current series developed de novo aneurysms or significant enlargement of previously untreated aneurysms between 6 months and 12 years after initial presen- tation (Table 2) underscores the need for careful follow-up. The only death in the series was attributable to a de novo aneurysm in an HIV-positive patient who had an SAH before the new aneurysm had been discovered.

$\mathrm{SAH}$ is generally considered better tolerated by children than by adults, though the mechanism for this better tolerance is uncertain. Several series have reported the low rate of clinically significant vasospasm in cases of ruptured pediatric intracranial aneurysms. ${ }^{18,20,22}$ Our series also confirms this-4 patients had imaging-proved vasospasm and only 1 required therapy to reverse an ischemic neurologic deficit. The basis for children's better tolerance of vasospasm, a leading cause of morbidity in adult patients with $\mathrm{SAH}$, warrants further investigation.

Aneurysm retreatment is an important feature in our cohort. During an average 2-year follow-up period, 4/19 (21\%) patients treated by selective coil embolization required retreatment of the index aneurysm. In this same group, 2/19 $(10 \%)$ crossed over to microneurosurgical treatment. Given the cumulative nature of aneurysm recanalization and evidence of significantly delayed recanalization in children ( $\leq 6$ years in this study), it is possible that longer follow-up would reveal a higher proportion of treatment failure. By the same token, 1/19 (5\%) patients treated with incomplete selective clipping crossed over to coil embolization and 3/10 (30\%) patients initially receiving bypass/trapping crossed over to endovascular parent artery occlusion (1 patient) or selective 


\begin{tabular}{|c|c|c|c|c|}
\hline Series & Current & Lasjaunias et $\mathrm{al}^{18}$ & Huang et $\mathrm{al}^{22}$ & Agid et $\mathrm{al}^{29}$ \\
\hline Patients (No.) & 77 (103 aneurysms) & 59 (75 aneurysms) & 706 & 33 (37 aneurysms) \\
\hline \multicolumn{5}{|l|}{ Etiology/morphology } \\
\hline Fusiform & $31 \%$ & $56 \%$ & - & $19 \%$ \\
\hline Saccular & $46 \%$ & $27 \%$ & - & $46 \%$ \\
\hline Infectious & $12 \%$ & $14 \%$ & - & $8 \%$ \\
\hline Traumatic & $14 \%$ & $3 \%$ & - & $14 \%$ \\
\hline Giant (>25 mm) & $11 \%$ & - & $20 \%$ & - \\
\hline Multiple & $16 \%$ of patients, $38 \%$ of aneurysms & $15 \%$ & - & - \\
\hline Posterior circulation & $22 \%$ & $27 \%$ & $17 \%$ & $24 \%$ \\
\hline Age & $12 \mathrm{yr}(3 \mathrm{mo}$ to $18 \mathrm{y})$ & $7.6 \mathrm{yr}$ (8 days to $15 \mathrm{yr}$ ) & $0-18 \mathrm{yr}$ & 10.2 y (1 day to $17 \mathrm{yr})$ \\
\hline Sex (\% male) & $48 \%$ & $59 \%$ & $63 \%$ & $48 \%$ \\
\hline Hemorrhage & $32 \%$ & $54 \%$ & $80 \%$ & $27 \%$ \\
\hline Mortality & $1.3 \%$ & $10.4 \%$ & $28 \%$ & $15 \%$ \\
\hline
\end{tabular}

Note:- - indicates not applicable.

coiling ( 1 for the index aneurysm and 1 for a de novo aneurysm). The only fatality in the series occurred due to rupture of a de novo aneurysm as opposed to rupture or rerupture of an index aneurysm.

Although a recent study ${ }^{26}$ of adult patients who had undergone therapeutic carotid occlusions found that no new aneurysms developed during a mean surveillance of 4 years and another study ${ }^{27}$ described a $1.5 \%$ incidence of de novo aneurysms at screening MR angiography (MRA) 5 years following index aneurysm coiling in adults, a third recent article reported that $10 \%$ of unruptured intracranial aneurysms in adults underwent enlargement during 4 years of MRA followup. ${ }^{28}$ Other large series of endovascular and surgical treatments of pediatric aneurysms ${ }^{18,29}$ have had, like the current study, relatively limited follow-up (particularly considering the long lives ahead of children); hence, the issue of index and nontarget aneurysm treatment and retreatment bears further elucidation, with better long-term imaging surveillance.

Treatment-related morbidity and mortality were low in both endovascular and surgical groups compared with rates in the medical literature (Table 3). Complication rates generally decreased with time, with the only death occurring in 1991 and 5 of 6 documented symptomatic infarctions occurring before 2000. The overall infarction rate was higher following surgical treatment $(4 / 29$ or $14 \%)$ than following endovascular treatment $(2 / 30$ or $7 \%)$. Ventriculitis (2/29 or $7 \%)$ and development of seizures (3/29 or $10 \%)$ were also noted postsurgically but not following endovascular treatment. Selection bias may play a role in these complication rates, because not all aneurysms were equally amenable to endovascular compared with surgical treatment. No definite delayed sequelae of $\mathrm{x}$-ray radiation exposure for endovascular therapy were documented, but the short average follow-up period limits the utility of this observation.

As in any longitudinal case series, modes of treatment have evolved significantly during the course of the study. Microsurgical and endovascular techniques have both advanced during the past nearly 3 decades, as has intensive care for critically ill children. Technical complications early in the series are less instructive for management of future patients than are data regarding the need for long-term follow-up of patients regardless of treatment technique. As demonstrated in Fig 4, many patients in this series received both endovascular and surgical therapies. Crossover between treatment modalities makes drawing conclusions about the efficacy of either alone difficult. Such complex cases do, however, underscore the importance of having both surgical and endovascular techniques available for the management of pediatric aneurysms. Intracranial aneurysms in children are heterogeneous and present management challenges that require participation of a multidisciplinary team for optimal care.

\section{Conclusions}

This constitutes the largest single-institution series of pediatric intracranial aneurysms (excluding those associated with brain arteriovenous malformations) to date. Fewer than half of patients in this group had comorbidities or preconditions thought to be associated with aneurysm formation. The current study confirms a higher rate of posterior circulation and fusiform aneurysms as compared with adult patients with aneurysms. This study also demonstrates improved periprocedural and short-term outcomes compared with those reported in prior surgical and endovascular-treatment series. These likely reflect continuous improvements during the past 3 decades in endovascular and microsurgical techniques for aneurysm treatment and improved early diagnosis of aneurysms and optimization of intensive care for pediatric patients. A significant caveat, however, is that the follow-up term after initial aneurysm treatment averaged only 4 years, thus limiting the ability to detect development of subsequent aneurysms or other long-term manifestations of aneurysmal disease. Despite the relatively short follow-up period, $8 \%$ of patients developed new or expanding aneurysms warranting additional treatment (Table 2). Although some of these new aneurysms may have formed due to increased wall stresses on inherently dysplastic vessels in the setting of increased flow through remaining vessels after occlusion of a carotid or vertebral artery (eg, Fig 6), other aneurysms grew despite preservation of parent artery flow (eg, Fig 5). New and enlarging aneurysms developed in patients initially treated surgically and endovascularly, as has been reported previously by other investigators. ${ }^{30}$ In sum, these findings suggest that a brain aneurysm in a child should be considered as a potentially chronic progressive condition. Vigilant long-term follow-up with appropriate clinical and minimally invasive imaging surveillance appears warranted and prudent. 


\section{References}

1. Gerosa M, Licata C, Fiore DL, et al. Intracranial aneurysms of childhood. Childs Brain 1980;6:295-302

2. Meyer FB, Sundt TM Jr, Fode NC, et al. Cerebral aneurysms in childhood and adolescence. J Neurosurg 1989;70:420-25

3. Ostergaard JR, Voldby B. Intracranial arterial aneurysms in children and adolescents. J Neurosurg 1983;58:832-37

4. Patel AN, Richardson AE. Ruptured intracranial aneurysms in the first two decades of life: a study of $\mathbf{5 8}$ patients. J Neurosurg 1971;35:571-76

5. Roche JL, Choux M, Czorny A, et al. Intracranial arterial aneurysm in children: a cooperative study apropos of $\mathbf{4 3}$ cases [in French]. Neurochirurgie 1988; 34:243-51

6. Sedzimir CB, Robinson J. Intracranial hemorrhage in children and adolescents. J Neurosurg 1973;38:269-81

7. Pasqualin A, Mazza C, Cavazzani $P$, et al. Intracranial aneurysms and subarachnoid hemorrhage in children and adolescents. Childs Nerv Syst 1986;2: 185-90

8. Locksley HB, Sahs AL, Sandler R. Report on the cooperative study of intracranial aneurysms and subarachnoid hemorrhage. 3. Subarachnoid hemorrhage unrelated to intracranial aneurysm and A-V malformation: a study of associated diseases and prognosis. J Neurosurg 1966;24:1034-56

9. Allison JW, Davis PC, Sato Y, et al. Intracranial aneurysms in infants and children. Pediatr Radiol 1998;28:223-29

10. Amacher AL, Drake CG, Ferguson GG. Posterior circulation aneurysms in young people. Neurosurgery 1981;8:315-20

11. Heiskanen $O$, Vilkki J. Intracranial arterial aneurysms in children and adolescents. Acta Neurochir (Wien) 1981;59:55-63

12. Proust F, Toussaint P, Garnieri J, et al. Pediatric cerebral aneurysms. J Neurosurg 2001;94:733-39

13. Juvela S. Risk factors for multiple intracranial aneurysms. Stroke 2000;31:392-97

14. Juvela S. Natural history of unruptured intracranial aneurysms: risks for aneurysm formation, growth, and rupture. Acta Neurochir Suppl 2002;82:27-30

15. King JT Jr. Epidemiology of aneurysmal subarachnoid hemorrhage. Neuroimaging Clin N Am 1997;7:659-68

16. Rinkel GJ, Djibuti M, Algra A, et al. Prevalence and risk of rupture of intracranial aneurysms: a systematic review. Stroke 1998;29:251-56
17. Lasjaunias P. From aneurysm to aneurysmal vasculopathies. Interv Neuroradiol 2000;5:105-08

18. Lasjaunias $\mathrm{P}$, Wuppalapati S, Alvarez $\mathrm{H}$, et al. Intracranial aneurysms in children aged under 15 years: review of 59 consecutive children with 75 aneurysms. Childs Nerv Syst 2004;21:437-50

19. terBrugge KG. Neurointerventional procedures in the pediatric age group. Childs Nerv Syst 1999;15:751-54

20. Aryan HE, Giannotta SL, Fukushima T, et al. Aneurysms in children: review of 15 years experience. J Clin Neurosci 2006;13:188-92

21. Herman JM, Rekate HL, Spetzler RF. Pediatric intracranial aneurysms: simple and complex cases. Pediatr Neurosurg 1991;17:66-72, discussion 73

22. Huang J, McGirt MJ, Gailloud P, et al. Intracranial aneurysms in the pediatric population: case series and literature review. Surg Neurol 2005;63:424-32, discussion 432-33

23. Sanai N, Quinones-Hinojosa A, Gupta NM, et al. Pediatric intracranial aneurysms: durability of treatment following microsurgical and endovascular management. J Neurosurg 2006;104:82-89

24. Molyneux A, Kerr R, Stratton I, et al. International Subarachnoid Aneurysm Trial (ISAT) of neurosurgical clipping versus endovascular coiling in 2143 patients with ruptured intracranial aneurysms: a randomised trial. Lancet 2002;360:1267-74

25. Bulsara KR, Raja A, Owen J. HIV and cerebral aneurysms. Neurosurg Rev 2005;28:92-95

26. de Gast AN, Sprengers ME, van Rooij WJ, et al. Long-term 3T MR angiography follow-up after therapeutic occlusion of the internal carotid artery to detect possible de novo aneurysm formation. AJNR Am J Neuroradiol 2007;28: 508-10

27. Sprengers ME, van Rooij WJ, Sluzewski M, et al. MR angiography follow-up 5 years after coiling: frequency of new aneurysms and enlargement of untreated aneurysms. AJNR Am J Neuroradiol 2009;30:303-07

28. Burns JD, Huston J, Layton KF, et al. Intracranial aneurysm enlargement on serial magnetic resonance angiography: frequency and risk factors. Stroke 2009;40:406-11

29. Agid R, Souza MPS, Reintamm G, et al. The role of endovascular treatment for pediatric aneurysms. Childs Nervous System 2005;21:1030-36

30. Agid R, Terbrugge K. Pediatric aneurysms. J Neurosurg 2007;106(4 supple):328, author reply $328-29$ 